\title{
Cobalt and Nickel Nanoparticles Binary Catalyst Modified Glassy Carbon Electrode for Glucose and Ascorbic Acid Electrooxidation in Alkaline Medium
}

\author{
Mohamed I. Awad ${ }^{1,2, *}$, B. A. Al-Jahdalyl, Omar A. Hazazi ${ }^{1}$ and Mohammed A. Kassem ${ }^{1,3, *}$ \\ ${ }^{1}$ Chemistry Department, Faculty of Applied Sciences, Umm Al-Qura University, Makkah, Saudi \\ Arabia. \\ ${ }^{2}$ Chemistry Department, Faculty of Science, Cairo University, Cairo, Egypt. \\ ${ }^{3}$ Chemistry Department, Faculty of Science, Benha University, Benha13518, Egypt

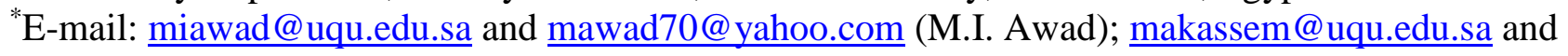 \\ maa_kassem@hotmail.com (M.A. Kassem)
}

doi: $10.20964 / 2020.10 .80$

Received: 6 April 2020 / Accepted: 25 May 2020 / Published: 30 November 2020

Glucose and ascorbic acid (AA) electrooxidation are examined at a binary catalyst of nickel and cobalt nanoparticles, modified in a certain order onto glassy carbon (GC) electrode. Either nano-Ni was deposited then followed by nano-Co or the reverse. The modified electrodes are characterized morphologically and voltammetrically. The electrochemical response of the nano-Co modified electrodes critically depends on the modification of the underlying substrate. Deposition of nano-Co on a pre-deposited nickel subjected to electrooxidation, designated as nano-Co/ $\mathrm{NiOx} / \mathrm{GC}$, resulted in a significant catalysis of glucose oxidation. The nano-Co/ $\mathrm{NiOx} / \mathrm{GC}$ electrode is utilized for the simultaneous investigation of glucose and AA using square wave voltammetry (SWV). The linear dynamic ranges for the two species were determined. The LOD and LOQ values were calculated and founded 0.176 and $0.528 \mu \mathrm{M}$ for AA and 0.066 and $0.199 \mu \mathrm{M}$ for glucose. Also, the precision of the developed method was evaluated by determining the relative standard deviation (RSD) and found less than $2.3 \%$.

Keywords: Binary electrocatalyst; Cobalt; Nickel; Nanoparticles; Glucose, Ascorbic acid; Voltammetry.

\section{FULL TEXT}

(C) 2021 The Authors. Published by ESG (www.electrochemsci.org). This article is an open access article distributed under the terms and conditions of the Creative Commons Attribution license (http://creativecommons.org/licenses/by/4.0/). 